PlastOx 2007 (2009) 179-189

(C) EDP Sciences, 2009

DOI: $10.1051 /$ ptox/2009013

\title{
Microstructure et chimie du substrat
}

\author{
R. Molins \\ École des Mines de Paris, ParisTech, Direction des Recherches, 60 Bld. Saint-Michel, \\ 75272 Paris Cedex 06, France \\ e-mail : regine.molins@ensmp.fr
}

\begin{abstract}
Résumé. Plusieurs exemples d'interactions microstructure - chimie - comportement des matériaux sont illustrés dans ce chapitre, à savoir l'influence du matériau et de sa microstructure sur les mécanismes d'oxydation et le type d'oxydes développés, inversement l'effet de l'oxydation sur les modifications microstructurales et chimiques du matériau et l'influence de la composition chimique (dont les éléments mineurs) du matériau sur la nature des couches oxydes et leur adhérence. Un exemple de ruine chimique dans le cas d'alliages FeCrAl sera présentée ainsi que certains effets mécaniques sur les phénomènes de croissance des couches.
\end{abstract}

\section{INTRODUCTION}

La résistance des matériaux à l'oxydation haute température est contrôlée par la qualité protectrice des couches d'oxydes qui les recouvrent. Pour cela, une optimisation du matériau est recherchée, en agissant sur sa microstructure et sa chimie, mais aussi au travers d'opérations de préparation ou de traitement de surface pour contrôler les propriétés des couches d'oxydes visées (adhérence, compacité, conductibilité....). Si la durée de vie face à l'oxydation d'une structure donnée est généralement associée au caractère protecteur des couches développées (caractère d'inoxydabilité par exemple), les modes de croissance des couches engendrent des modifications dans le substrat sous-jacent, citons l'appauvrissement en éléments d'alliage qui peut conditionner le comportement mécanique ultérieur de la zone affectée, ou l'introduction de défauts conduisant à un endommagement prématuré de la structure. Si les critères d'optimisation, en termes de composition chimique et de microstructure, aptes à assurer le développement d'une couche protectrice $\left(\mathrm{Cr}_{2} \mathrm{O}_{3}, \mathrm{Al}_{2} \mathrm{O}_{3}\right)$ ont été largement étudiées, les échelles de perturbation (de quelques nanomètres à plusieurs dizaines de microns) résultantes dans le substrat sont moins détaillées dans la littérature, bien que ces zones affectées consécutivement aux phénomènes d'oxydation puissent modifier le comportement ultérieur d'une structure donnée et porter atteinte à sa durée de vie.

Les effets induits dans le matériau peuvent présenter un caractère adoucissant (dissolution de phase, transformation de phase, cavitation...) ou renforçant (enrichissement en interstitiel, oxydation interne, dispersoïde d'oxydes... ).

Nous traiterons dans ce chapitre de plusieurs effets de corrélation matériau/oxydation, à savoir l'influence de la microstructure du matériau (taille de grains, phases, cristallographie) sur les couches d'oxydes développées, l'effet de l'oxydation sur les modifications microstructurales et chimiques du matériau puis l'influence de la chimie du matériau sur les couches oxydes (teneur en éléments d'alliages $(\mathrm{Cr}, \mathrm{Al})$, impuretés (mineurs, S), éléments actifs). Un exemple de ruine chimique d'une structure sera détaillé ainsi que certains effets mécaniques.

Les matériaux servant de fils conducteurs sont essentiellement des alliages commerciaux, et couvrent la familles des alliages à base de nickel (Ni, Ni-Cr, superalliages), ainsi que des alliages $\mathrm{FeCrAl}$ et $\mathrm{Ni}$ - $\mathrm{Al}$.

Compte tenu des échelles auxquelles se produisent les phénomènes mis en jeu, les études de caractérisation ont souvent été menées en microscopie électronique en transmission, excellent moyen d'investigation pour étudier finement les processus d'oxydation. La microscopie électronique en 


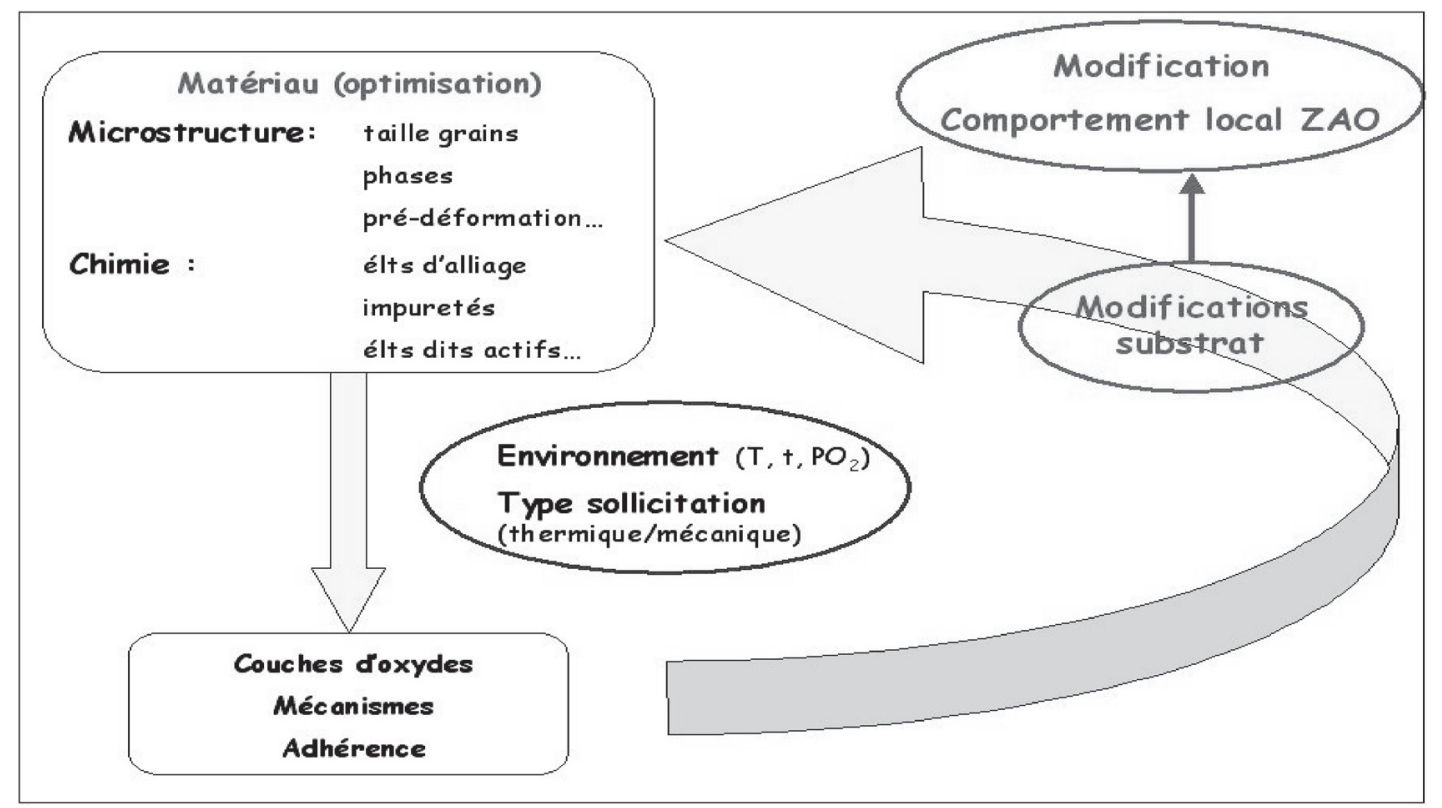

Figure 1. Synoptique matériau/oxydation.

transmission permet en effet d'une part de suivre les processus de germination des oxydes en fonction du matériau (microstructure, chimie, état de surface) et des conditions environnementales (température, temps, environnement) et d'autre part d'identifier la nature et la morphologie des oxydes formés ainsi que les modifications résultantes dans le matériau, en fonction des conditions de sollicitations thermiques/mécaniques.

La figure 1 schématise les paramètres matériau (microstructure et chimie) à prendre en compte pour satisfaire à une oxydation contrôlée tout en rappelant que tout phénomène de corrosion haute température influe réciproquement sur le matériau en engendrant des perturbations locales qui pourront engendrer des modifications du comportement mécanique de la zone affectée par l'oxydation.

\section{INFLUENCE DE LA MICROSTRUCTURE DU SUBSTRAT SUR L'OXYDATION}

Dès les premiers stades d'oxydation, le nombre et la forme des germes d'oxydes qui se développent en surface d'un matériau dépendent fortement de l'état de surface, du degré de pureté, de l'orientation cristalline du matériau et des conditions expérimentales. Dans le cas du nickel, où le système $\mathrm{Ni} / \mathrm{NiO}$ est considéré comme modèle (les deux réseaux sont cubiques à faces centrées et l'écart entre les paramètres de maille est de 18\%), des observations du stade de germination montrent un comportement différent en fonction de l'orientation cristalline du grain sous-jacent. Un film très mince monocristallin peut se former sur certains grains alors que l'oxyde apparaît polycristallin et épais sur d'autres grains. La comparaison de l'oxydation de monocristaux orientés permet de classer les cinétiques d'oxydation en fonction de l'orientation cristalline comme suit : $(112)<(111)<(100)$. L'oxydation rapide de la face (100) du nickel est attribuée à la formation d'un oxyde polycristallin, avec de petits grains et un grand nombre de sous-joints, agissant comme courts-circuits de diffusion pour la migration des cations $\mathrm{Ni}^{2+}$ vers la surface. A l'inverse, une face (112) du nickel s'oxyde lentement en raison de la formation d'un oxyde présentant une orientation topotactique avec le substrat, et des sous-joints faiblement désorientés [1-3]. 
Pour les alliages Ni-Cr, des germes de $\mathrm{NiO}$ et $\mathrm{Cr}_{2} \mathrm{O}_{3}$ se développent simultanément à la surface du matériau. $\mathrm{Si} \mathrm{Cr}_{2} \mathrm{O}_{3}$ est plus stable thermodynamiquement que $\mathrm{NiO}$, la croissance de $\mathrm{NiO}$ est néanmoins plus rapide et cet oxyde tend à recouvrir rapidement les germes de $\mathrm{Cr}_{2} \mathrm{O}_{3}$. Si la teneur en chrome de l'alliage est suffisante, les germes de $\mathrm{Cr}_{2} \mathrm{O}_{3}$ peuvent croître latéralement et conduire à une couche interne continue ; dans le cas contraire, les germes se retrouvent dispersées dans $\mathrm{NiO}$. La formation d'une couche de $\mathrm{Cr}_{2} \mathrm{O}_{3}$ est favorisée lorsque la teneur en chrome augmente, la taille de grains de l'alliage diminue, l'écrouissage augmente ou quand la pression d'oxygène diminue $[4,5]$.

Les interfaces (joints de grains, interphases) jouent un rôle particulier, en favorisant la diffusion du chrome ou des éléments d'alliage qui s'y concentrent. Pour les alliages $\mathrm{Ni}-\mathrm{Cr}$, quelle que soit la teneur en chrome, les joints de grains se tapissent d'îlots discontinus de $\mathrm{Cr}_{2} \mathrm{O}_{3}$. Ces îlots de $\mathrm{Cr}_{2} \mathrm{O}_{3}$ coalescent avec le temps jusqu'à former un film continu, le long duquel s'étend une zone peu oxydée et appauvrie en chrome [3].

\section{EFFET DE L'OXYDATION SUR LES MODIFICATIONS MICROSTRUCTURALES ET CHIMIQUES DU MATÉRIAU}

La croissance des couches d'oxydes s'accompagne de modifications structurales et chimiques du substrat susceptibles d'influer sur le comportement local de la zone affectée par l'oxydation sous la surface oxydée. Les exemples qui suivent rendent compte de ce phénomène, consécutif à des processus de diffusion d'éléments d'alliage, de transformations de phase et d'injection de défauts.

Pour les alliages $\mathrm{Ni}-\mathrm{Cr}$, les couches d'oxyde et les mécanismes mis en jeu diffèrent en fonction de la teneur en chrome et de la taille de grains [6]. Si pour de faibles teneurs en chrome, la couche de $\mathrm{Cr}_{2} \mathrm{O}_{3}$ ne peut recouvrir la surface de l'alliage et conduit à de l'oxydation interne, une teneur élevée permet la formation d'une couche continue de $\mathrm{Cr}_{2} \mathrm{O}_{3}$ à la surface du matériau. Pour des teneurs intermédiaires entre 10 et $30 \%, \mathrm{Cr}_{2} \mathrm{O}_{3}$ se forme préférentiellement à l'aplomb des joints de grains alors que $\mathrm{NiO}$ croît sur le reste de la surface. La formation d'une couche continue de $\mathrm{Cr}_{2} \mathrm{O}_{3}$ s'obtient par croissance latérale à partir des joints de grains sous la couche externe de NiO. L'établissement de ces deux couches est fortement influencé par la taille des grains de l'alliage et l'état métallurgique de départ (écrouissage de surface). Plus la taille de grain est petite, plus la formation de la couche continue de $\mathrm{Cr}_{2} \mathrm{O}_{3}$ sera rapide [7].

L'oxydation à $650^{\circ} \mathrm{C}$ sous air d'alliages Ni-Cr à respectivement 20 et $30 \%$ de chrome montre que l'alliage contenant $20 \%$ en poids de chrome forme dès le début de l'oxydation un oxyde de type $\mathrm{NiO}$ avant la mise en place d'une sous-couche de $\mathrm{Cr}_{2} \mathrm{O}_{3}$. Une zone d'oxydation interne de $\mathrm{Cr}_{2} \mathrm{O}_{3}$ dans le substrat s'étend sous la surface oxydée sur une distance qui augmente avec le temps d'oxydation. Ces précipités de $\mathrm{Cr}_{2} \mathrm{O}_{3}$ se développent en relation d'épitaxie avec la matrice, avec les directions denses de la structure hexagonale compacte de $\mathrm{Cr}_{2} \mathrm{O}_{3}$ parallèles aux directions denses de la structure cubique à faces centrées de l'alliage de nickel. Aucun appauvrissement en chrome n'est détecté derrière la zone d'oxydation interne. Un alliage plus riche en chrome (30\% poids) forme dès le début une couche de $\mathrm{Cr}_{2} \mathrm{O}_{3}$ sans oxydation du nickel. En ce qui concerne les joints de grains, les deux alliages sont $\mathrm{Cr}_{2} \mathrm{O}_{3}$ formeurs à l'aplomb des joints avec indentation du joint.

Pour les deux alliages, la formation d'une couche continue d'oxyde de chrome s'accompagne d'une zone déchromisée dans l'alliage sous l'oxyde, qui entraîne une recristallisation de celle-ci et la formation de petits grains de taille très inférieure à la taille de grains initiale du matériau considéré [3]. Cette région de composition chimique proche d'un alliage $\mathrm{Ni}-\mathrm{Cr}$ à $10 \%$ en poids de chrome croît avec le temps d'oxydation. Plus l'épaisseur de la couche d'oxyde de chrome est élevée, plus la zone d'appauvrissement en chrome s'étend sur une distance importante. En l'absence de couche continue de $\mathrm{Cr}_{2} \mathrm{O}_{3}$, la matrice n'est pas appauvrie en chrome derrière la zone d'oxydation interne. Dès qu'une couche continue de $\mathrm{Cr}_{2} \mathrm{O}_{3}$ se forme, on observe une zone d'appauvrissement en chrome qui s'étend sous la surface oxydée sur une distance proportionnelle à l'épaisseur de la couche d'oxyde de chrome (figure 2). Cette zone modifiée aura une incidence sur les capacités de déformation d'un produit mince car plus le substrat 


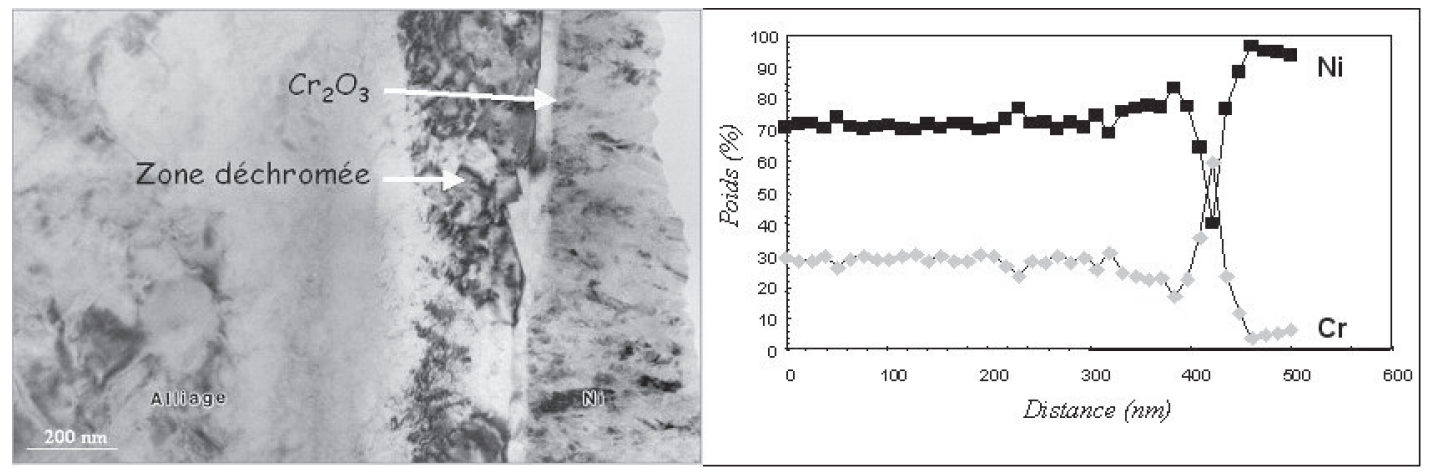

Figure 2. Ni-30 Cr oxydé 2 min à $650{ }^{\circ} \mathrm{C}$, corrélation $\mathrm{Cr}_{2} \mathrm{O}_{3}$ et zone appauvrie en $\mathrm{Cr}$ [3].

est mince, plus la relaxation des contraintes est importante. Le rapport entre l'épaisseur de la zone appauvrie en chrome et celle du substrat non perturbé est proche de 1 dans le cas d'un feuillard de $100 \mu \mathrm{m}$ d'épaisseur après 96 heures d'oxydation à $900^{\circ} \mathrm{C}$ et de l'ordre de $10^{-2}$ pour un échantillon massif de $1 \mathrm{~mm}$ d'épaisseur.

Dans le cas d'un superalliage à base de nickel monocristallin (AM1), constitué d'une matrice austénitique renforçée par des précipités ordonnés de phase $\gamma^{\prime}\left(\mathrm{Ni}_{3}(\mathrm{Ti}, \mathrm{Ta}, \mathrm{Al})\right)$, se présentant sous forme de cubes d'arête 0.5 micron, un traitement d'oxydation conduit à la disparition de la phase ordonnée durcissante sous la surface oxydée $[3,8]$. La formation de cette zone exempte de précipités résulte de la diffusion des éléments $\gamma$ '-gènes $(\mathrm{Al}, \mathrm{Ti})$ en surface pour former les couches d'oxydes. Cette zone, de même orientation cristalline que le matériau initial, présente une forte densité de dislocations, indiquant une importante déformation plastique lors de l'oxydation. L'extension de la zone affectée par l'oxydation croît avec la durée et la température du traitement thermique. Aux températures intermédiaires $\left(650^{\circ} \mathrm{C}\right)$, après une oxydation de 15 minutes [3], la zone appauvrie s'étend sur une centaine de nanomètres sous une couche d'oxyde duplex, constituée d'une strate externe de $\mathrm{NiO}$ et d'une strate interne identifiée comme un oxyde complexe $(\mathrm{Cr}, \mathrm{Ti}, \mathrm{Al})(\mathrm{Ta}, \mathrm{Ti}) \mathrm{O}_{4}$. A plus haute température $\left(110{ }^{\circ} \mathrm{C}\right)$, où se forme une couche interne d'alumine, la dissolution progressive de la phase ordonnée avec une modification de forme et de taille (cubique à sphérique) lorsqu'on se rapproche de la surface oxydée est aussi détectée en association avec une diminution de la teneur en aluminium [9] (figure 3).

Cet appauvrissement en phase durcissante apparaît aussi dans le cas d'un superalliage à base de nickel polycristallin, l'alliage 718 [3]. Le durcissement structural des grains de cet alliage est dû à la précipitation de deux phases intermétalliques, la phase $\gamma^{\prime}, \mathrm{Ni}_{3}$ ( $\mathrm{Ti}, \mathrm{Al}$ ) (précipités sphériques de diamètre variant de 20 à $50 \mathrm{~nm}$ ) et la phase $\gamma$ ", $\mathrm{Ni}_{3} \mathrm{Nb}$ (disques minces de diamètre 30 à $50 \mathrm{~nm}$ ). Après un traitement sous air de $15 \mathrm{~min}$ à $650^{\circ} \mathrm{C}$, deux couches d'oxydes se développent, une couche externe de $\mathrm{NiO}$ et une couche interne composée d'un mélange de $\mathrm{Cr}_{2} \mathrm{O}_{3}$ et de $\mathrm{CrNbO}_{4}$. Dans le même temps, apparaît sous la surface oxydée une région d'une centaine de nanomètres où les précipités durcissant la matrice ont disparu conjointement à un appauvrissement en chrome, niobium, titane et aluminium.

Ces effets de dissolution de phases dans le cas d'alliages renforcés par précipitation conduisent à un adoucissement local du matériau dans la zone affectée par l'oxydation dont le comportement mécanique sera modifiée. Ceci sera particulièrement sensible en pointe de fissures lors d'essais de fissuration ainsi que dans les zones relaxées sous l'oxyde lors d'essais de fluage sur produits minces.

Une modification de comportement mécanique (évaluée via la diminution des propriétés en traction) peut résulter de la formation de cavités sous les surfaces oxydées, consécutivement à un mécanisme de croissance cationique. Cet effet a été mis en évidence dans le cas de fibres métalliques d'alliage 601 , de diamètre 12 microns, lors de traitements d'oxydation de 30 min entre 650 et $900^{\circ} \mathrm{C}$ [10]. Les couches d'oxydes qui se développent sont constituées d'une couche externe de $\mathrm{NiO}$ et d'un spinelle 


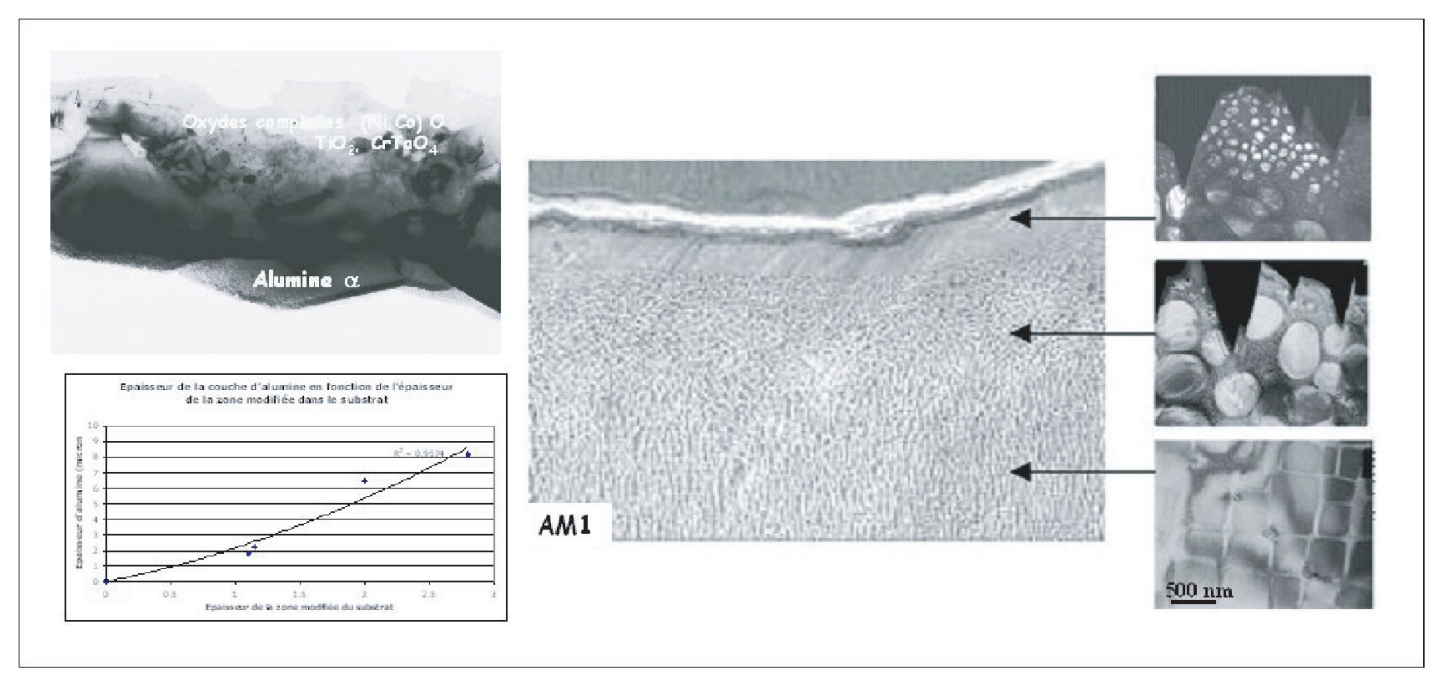

Figure 3. AM1 oxydé $10 \mathrm{~h}$ à $1100^{\circ} \mathrm{C}$, oxydes développés en surface du superalliage et dissolution des précipités $\gamma$ ' selon la distance à l'interface AM1/oxydes [9].

et d'une couche interne de $\mathrm{Cr}_{2} \mathrm{O}_{3}$. Sous la surface oxydée des fibres apparaissent des cavités facettées intragranulaires dont l'orientation est compatible avec la structure cubique de l'alliage et dont le nombre et la taille augmentent avec la température d'oxydation. La profondeur maximale d'apparition des cavités correspond à l'étendue de la zone appauvrie en chrome. L'origine de ces cavités est attribuée à un régime d'oxydation cationique responsable de l'injection de lacunes dans la fibre sous la surface oxydée. En effet, la croissance d'un oxyde par diffusion cationique entraîne la formation de lacunes qui peuvent être soit annihilées à l'interface métal-oxyde, soit injectées dans le substrat où elles sont soit annihilées sur des défauts (joints de grains, interfaces...) soit condensées sous forme de cavités. Pour une géométrie de type cylindrique (cas de la fibre), l'interface métal-oxyde est bloquée et ne peut servir de puits aux lacunes qui diffusent alors dans le substrat. De plus, pour des échantillons présentant un rapport surface sur volume élevé (tôles minces, fils), le nombre de puits disponibles dans l'alliage peut s'avérer insuffisant. Ce phénomène a été bien caractérisé lors de l'oxydation de fils de nickel de faible diamètre [11] et sur des lames minces oxydées in-situ où croissent des boucles de type lacunaire [12]. Dans le cas des fibres d'alliage 601 de faible diamètre et présentant une faible densité de défauts (peu de joints de grains et pas de précipités), les lacunes injectées se condensent sous forme de cavités dont la taille va croître avec le temps ou la température d'oxydation.

\section{INFLUENCE DE LA CHIMIE DU MATÉRIAU SUR LES COUCHES OXYDES}

Dans le cas du nickel, le taux d'impuretés influe notablement sur les mécanismes d'oxydation et la morphologie des couches de $\mathrm{NiO}$ développées [13]. Une étude menée sur deux grades de nickel pur $[14,15]$ montre, qu'après une oxydation de quelques heures à $800^{\circ} \mathrm{C}$, les deux nuances présentent un comportement différent (figure 4). Pour le nickel qualifié de très haute pureté, une unique couche de $\mathrm{NiO}$ de 5 microns d'épaisseur, composée de grains équiaxes (200 à $500 \mathrm{~nm}$ ) et contenant de nombreux pores, est observée. L'interface $\mathrm{Ni} / \mathrm{NiO}$ est facettée et aucune modification n'est détectée dans le métal. La croissance de la couche de $\mathrm{NiO}$ est associée à un mécanisme de diffusion essentiellement cationique. Dans le cas d'une nuance de nickel contenant $0.3 \%$ massique d'impuretés ( $\mathrm{Si}, \mathrm{Mn}, \mathrm{Mg}$, Ti), une couche duplex est formée, d'une épaisseur totale inférieure au cas précédent (2 à 3 microns). Cette couche se décompose en une couche externe de grains colonnaires d'environ $300 \mathrm{~nm}$ de largeur (formée selon 

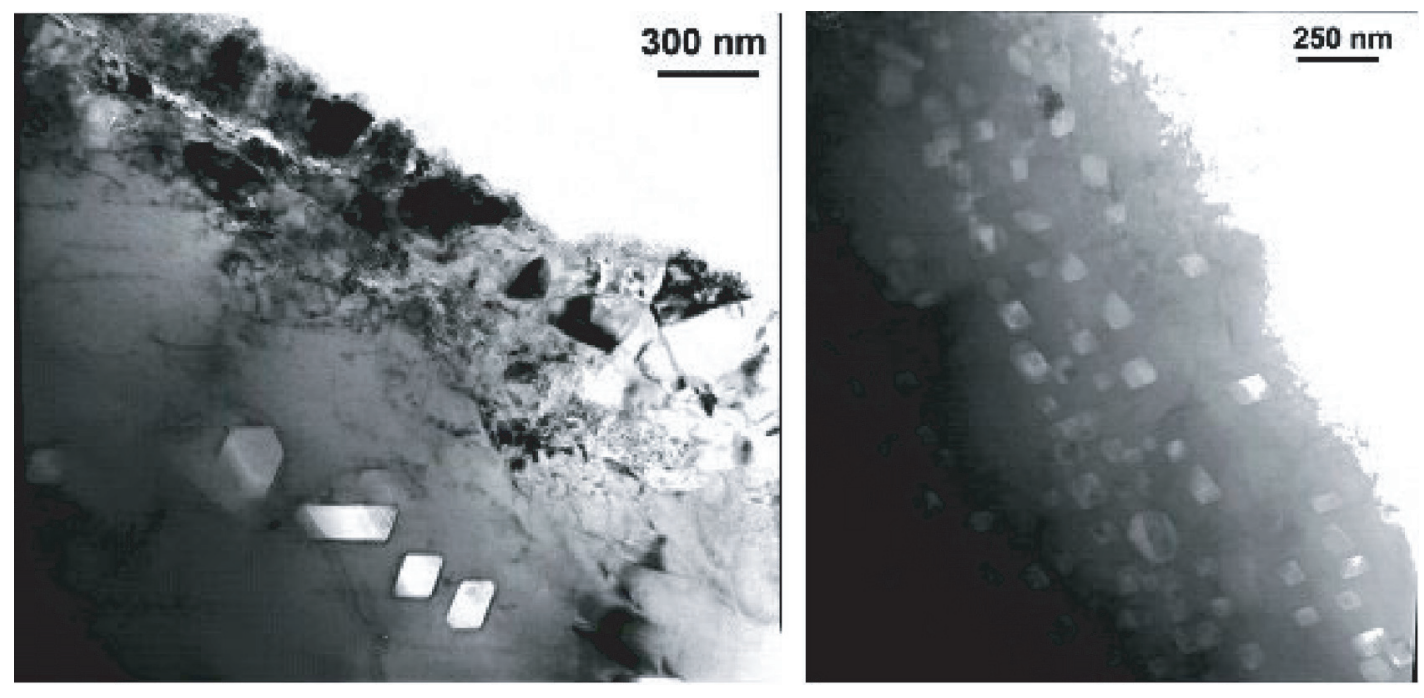

Figure 4. Composite Al/alliage 601, oxydé 30 min à $750^{\circ} \mathrm{C}$ [10].

un processus cationique) et d'une couche intermédiaire de petits grains équiaxes (formée selon un processus anionique). L'interface entre les deux strates est plane et correspond à la surface initiale du métal. L'interface entre le métal et la strate de $\mathrm{NiO}$ intermédiaire est par contre convolutée. Sous la couche oxydée, s'étend une zone affectée par l'oxydation avec de l'oxydation interne et de l'oxydation intergranulaire s'étendant sur plusieurs microns de profondeur. Le taux d'impuretés du nickel affecte par ailleurs la résistance à l'écaillage. Pour la nuance de très haute pureté, l'écaillage se produit à l'interface nickel/oxyde, mettant à nu la surface du métal. Pour la nuance contenant quelques impuretés, la résistance de l'interface $\mathrm{Ni} / \mathrm{NiO}$ interne est augmentée par un effet d'ancrage du à la présence de précipités interfaciaux et l'écaillage opère à l'interface $\mathrm{NiO}$ externe/ $\mathrm{NiO}$ interne.

Le soufre est une impureté inhérente aux alliages dont la diffusion et la ségrégation en surface durant un traitement d'oxydation à haute température dégradent la résistance de l'interface alliage/oxyde et conduisent à un écaillage prématuré de couches protectrices telles $\mathrm{Cr}_{2} \mathrm{O}_{3}$ et $\mathrm{Al}_{2} \mathrm{O}_{3}$, limitant ainsi la durabilité de certains composants industriels [16]. Cet effet soufre ainsi que sa distribution dans un système $\mathrm{Ni}-\mathrm{Al} / \mathrm{Al}_{2} \mathrm{O}_{3}$ ont été récemment montrés en comparant les couches d'alumine développées à $1100^{\circ} \mathrm{C}$ (alumine $\square$ ) sur deux alliages $\mathrm{Ni}-40 \mathrm{Al}$ (40\% massique en $\mathrm{Al}$ ), l'un ayant été dopé avec $40 \mathrm{ppm}$ de soufre [17]. Alors que le soufre ne modifie pas les mécanismes de transport dans la couche d'alumine, une accélération de la vitesse d'oxydation est observée pour l'alliage dopé en soufre et résulte d'une décohésion importante de zones oxydées, due à la coalescence de pores interfaciaux. De fait, l'action du soufre en excès dans l'alliage a une action directe sur le développement de cavités à l'interface dégradant l'adhérence de cette dernière. Dans le cas de l'alliage dopé en soufre, de nombreux précipités nanométriques, souvent associés à des pores, sont détectés dans la couche d'alumine duplex, avec des NiS dans la couche interne et $\mathrm{Ni}$ en couche externe. Seuls des précipités de nickel sont présents dans l'ensemble de la couche en l'absence d'excès de soufre. Outre la ségrégation de soufre détectée à l'interface alliage/oxyde, le soufre en excès peut donc être incorporé dans la couche d'alumine et précipiter sous forme de sulfures de nickel.

De nombreux articles décrivent le rôle bénéfique joué par les éléments actifs sur le comportement à l'oxydation des métaux et des alliages $(18,19,20)$. Parmi les effets bénéfiques, outre l'oxydation sélective de certains éléments et une cinétique d'oxydation ralentie $(21,22)$, nous pouvons citer l'augmentation de l'adhérence de la couche d'oxyde, la modification du mécanisme de croissance 


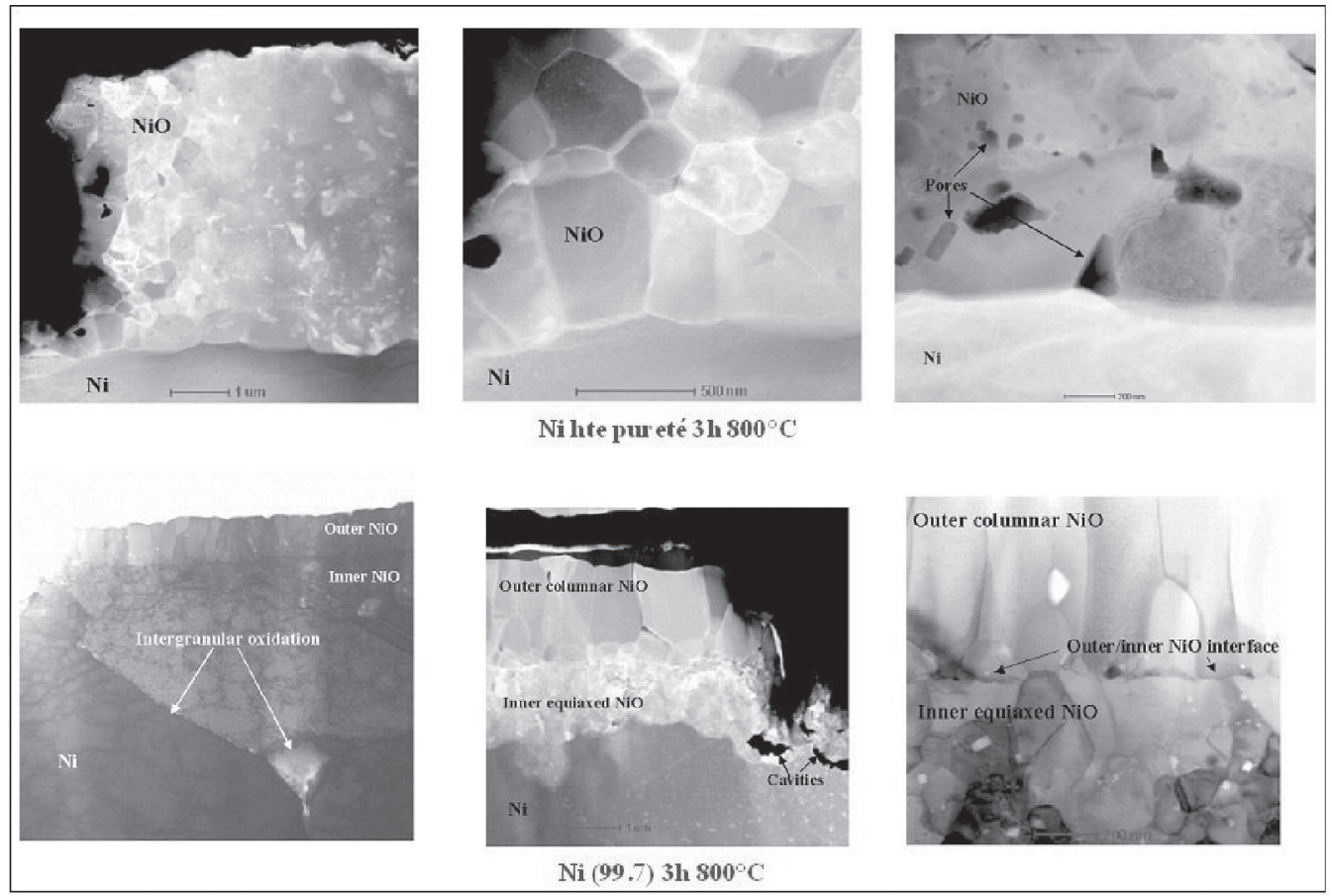

Figure 5. Influence de la pureté du nickel sur les couches de $\mathrm{NiO}$ [14].

(passage d'un mode cationique à un mode anionique) et la modification de la morphologie des grains d'oxyde (diminution de la taille de grains).

Pour les alliages alumino-formeurs dopés en éléments actifs (éléments des terres rares introduits en faible quantité), sous forme métallique ou dispersoïde d'oxydes en quantité contrôlée [23], la couche d'alumine $\alpha$ qui se développe à haute température est plus compacte et adhérente au substrat. Les alliages renforcés par dispersion d'oxydes (ODS) allient, grâce à l'ajout du dispersoïde, une bonne résistance mécanique et une bonne résistance à l'oxydation [24]. Les couches d'alumine $\alpha$ développées à des températures supérieures à $1000^{\circ} \mathrm{C}$ présentent une morphologie duplex avec une large couche de grains colonnaires surmontée d'une fine couche de grains équiaxes à l'interface gaz. La couche colonnaire est très compacte et adhérente au substrat. L'interface alliage/oxyde présente un faciès facetté et montre un enrichissement en titane, de plus en plus prononcé avec la durée d'oxydation, sous forme de carbure TiC (présence de particules isolées jusqu'à un film continu pour des durées longues d'oxydation). Les particules d'yttrine se trouvent uniformément dispersées dans l'alliage et de l'yttrium ségrégé est détecté à l'interface alliage/alumine de part et d'autre du liséré de TiC ainsi qu'aux joints de grains colonnaires de l'alumine.

\section{CONCEPTION ET RUINE}

Si l'ajout de particules d'yttrine à un alliage $\mathrm{FeCrAl}$ est bien connu pour améliorer la qualité protectrice de la couche d'alumine qui se forme à haute température ainsi que sa résistance au fluage, lors de sollicitations longue durée en conditions de service de produits minces, les ruptures et nombreux écaillages de la couche d'alumine entraînent une diminution du taux d'aluminium du substrat et en dessous d'une teneur critique, l'alliage ne peut plus former de couche protectrice [25, 26]. S'amorce alors un régime d'oxydation catastrophique conduisant rapidement à la destruction du matériau. Les 

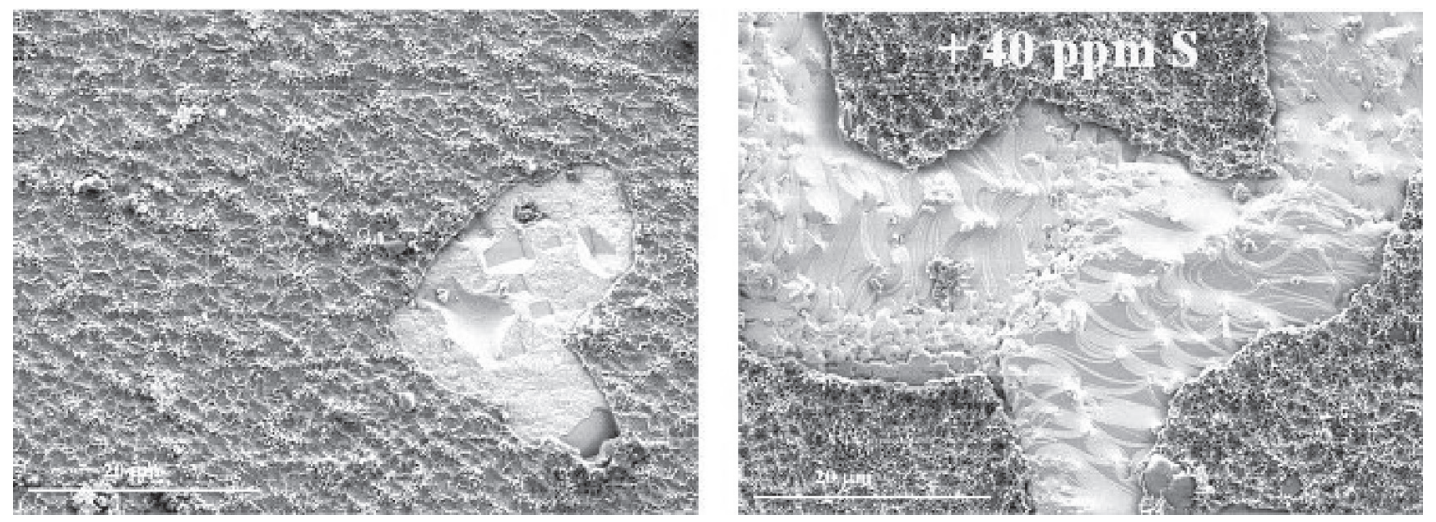

Figure 6. Effet du soufre sur l'écaillage de $\mathrm{Ni}-40 \mathrm{Al}$ oxydé 1 h à $1100^{\circ} \mathrm{C}$ [17].

différentes étapes conduisant à l'oxydation catastrophique (ruine chimique du matériau) sont les suivantes :

- un régime d'oxydation stable au cours duquel une couche d'alumine continue croît en surface entraînant une diminution progressive de la teneur en aluminium de l'alliage :

- un régime transitoire lorsque la teneur en aluminium de l'alliage atteint la valeur critique en dessous de laquelle une couche protectrice d'alumine ne peut plus se former (transition oxydation externe/oxydation interne). L'azote diffuse alors via les défauts de la couche d'alumine et sa diffusion dans le matériau étant plus rapide que celle de l'oxygène conduit à la formation de précipités d'AlN entraînant une accélération de la consommation de l'aluminium de la matrice jusqu'à l'épuisement du réservoir d'aluminium.

- un régime d'oxydation catastrophique: l'oxygène réagit avec le matériau dépourvu d'aluminium pour former de l'oxyde de chrome puis des oxydes de fer. A la fin de ce régime, l'alliage s'est entièrement transformé en une "éponge d'oxydes", avec une couche superficielle d'oxyde de fer et des oxydes mixtes de fer et de chrome à cœur.

De nombreux modèles de prévision de durée de vie pour produits minces ont été développés en fonction de paramètres matériau (réservoir aluminium, géométrie) et des conditions de sollicitation.

\section{EFFETS MÉCANIQUES}

La cinétique de formation d'une couche protectrice est fortement dépendante du mode de germination et de l'état métallurgique du matériau. Ainsi, la protection de la surface se produit d'autant plus facilement que le taux de pré-déformation est élevé. Ce phénomène exploitable industriellement est en général indissociable de l'état de contrainte résiduel (compression) consécutif au traitement de surface. Une pré-déformation résultant d'une mise en forme à froid (laminage, étirage) ou d'un traitement de surface (sablage, billage) modifie les mécanismes d'oxydation en favorisant l'oxydation sélective de certains éléments. La déformation de la région sous la surface de l'alliage, en créant des courts-circuits de diffusion et en augmentant la densité de défauts, modifie la cinétique et le mode d'oxydation. Cet effet est relativement bien connu dans le cas de la formation préférentielle d'une couche de $\mathrm{Cr}_{2} \mathrm{O}_{3}$ sur les alliages ne contenant pas suffisamment de chrome pour parvenir à se protéger par une couche de ce type sans pré-déformation. Une pré-déformation mécanique favorise l'oxydation sélective du chrome à la surface d'un alliage $\mathrm{Ni}-20 \mathrm{Cr}$, alors que les deux oxydes $\mathrm{NiO}$ et $\mathrm{Cr}_{2} \mathrm{O}_{3}$ sont identifiés simultanément pour un échantillon non déformé $[6,27]$.

L'accélération de la protection de la surface par une déformation mécanique a aussi été confirmée dans le cas de superalliages à base de nickel. Des observations à l'échelle de la microscopie électronique 

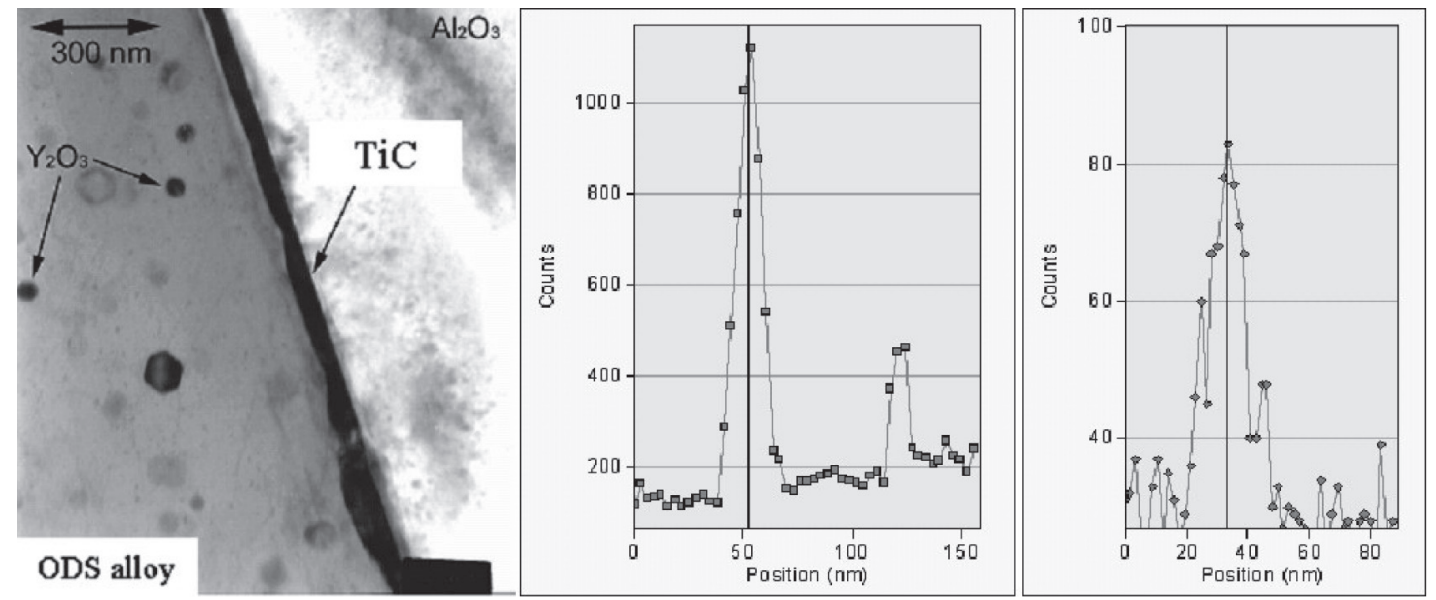

Figure 7. Alliage ODS oxydé 15 jours à $1300^{\circ} \mathrm{C}$, ségrégation d'Y à l'interface alliage/alumine et dans les joints de grains de l'alumine.

en transmission du stade de germination des oxydes montrent une oxydation préférentielle du chrome à l'aplomb des joints de grains, des joints de macles et à l'émergence des bandes de déformation dans le cas de l'alliage $\mathrm{Ni}-20 \mathrm{Cr}$ ou de l'alliage 718 pré-déformé [3]. Ces observations sont en accord avec les observations d'oxydation in situ [28], révélant une oxydation préférentielle à l'aplomb des joints de grains mais aussi joints de macles de recuit et des émergences des bandes de déformation.

Des traitements d'oxydation sous contrainte imposée en fluage tension sur des feuillards de $200 \mu \mathrm{m}$ de l'alliage Ni-20Cr, ont permis de suivre l'effet d'une sollicitation mécanique sur le mécanisme (nature, morphologie des couches, réactions interfaciales) et la cinétique d'oxydation. L'évolution des couches d'oxydes a été suivie en fonction du niveau de contrainte imposée et de la durée de l'essai, pour deux températures $600^{\circ} \mathrm{C}$ et $900^{\circ} \mathrm{C}$, et comparée à des essais d'oxydation statique, soit sans sollicitation externe $[29,30]$.

En l'absence de sollicitation mécanique, la couche d'oxyde se développe sous forme de festons ancrés aux joints de grains du substrat. A $600^{\circ} \mathrm{C}$, la teneur initiale du chrome dans l'alliage est insuffisante pour former une couche continue de $\mathrm{Cr}_{2} \mathrm{O}_{3}$ et la cinétique d'oxydation est contrôlée par la croissance de $\mathrm{NiO}$. La couche oxydée est constituée d'une strate externe de $\mathrm{NiO}$ et d'une couche intermédiaire composée d'un mélange d'oxydes, $\mathrm{NiO}, \mathrm{Cr}_{2} \mathrm{O}_{3}$ et $\mathrm{NiCr}_{2} \mathrm{O}_{4}$. La diffusion de l'oxygène dans le substrat conduit à la formation d'une zone d'oxydation interne caractérisée par des particules de $\mathrm{Cr}_{2} \mathrm{O}_{3}$ dans une matrice appauvrie en chrome. A $900{ }^{\circ} \mathrm{C}$, une couche continue de $\mathrm{Cr}_{2} \mathrm{O}_{3}$ se forme rapidement sous une couche externe de $\mathrm{NiO}$ et une couche intermédiaire de $\mathrm{NiCr}_{2} \mathrm{O}_{4}$. Deux stades d'oxydation sont identifiés, le premier étant dû à la croissance de $\mathrm{NiO}$, avant la formation d'une couche continue d'oxyde de chrome.

Sous l'application d'une contrainte mécanique, la nature des couches n'est pas modifiée mais une accélération de la croissance de certaines couches apparaît en fonction des conditions de sollicitation.

A $600^{\circ} \mathrm{C}$, les essais réalisés pour de courtes durées d'oxydation et de faibles taux de déformation ne montrent aucune différence. Toutefois, quand le niveau de déformation dépasse une valeur critique, de nombreux défauts (cavités, fissures ouvertes) sont crées dans la couche de $\mathrm{NiO}$, leur nombre augmentant avec le temps ou la contrainte. Ces défauts en agissant comme courts-circuits de diffusion pour l'oxygène vont modifier les mécanismes et conduire à une accélération de la croissance de $\mathrm{NiO}$ et une progression plus rapide du front d'oxydation interne (figure 7).

A $900^{\circ} \mathrm{C}$, l'application d'une contrainte montre que la croissance de la couche intermédiaire de $\mathrm{NiCr}_{2} \mathrm{O}_{4}$ est accélérée pour les temps courts, alors que pour des temps plus longs ou sous contrainte 


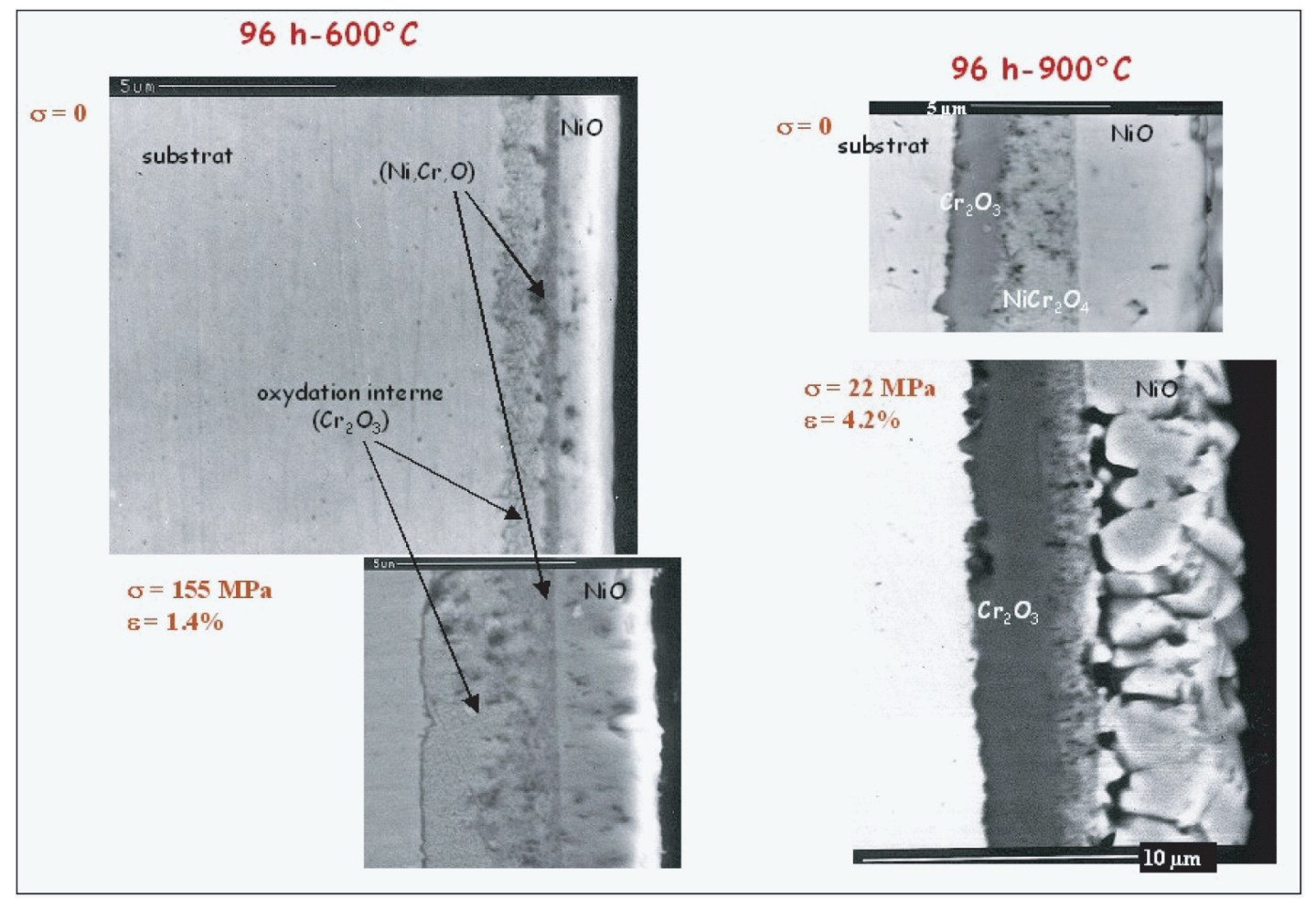

Figure 8. Influence de la contrainte sur les couches d'oxydes après $96 \mathrm{~h}$ à $600^{\circ} \mathrm{C}$ et $900^{\circ} \mathrm{C}$ [30].

élevée, c'est la croissance de $\mathrm{Cr}_{2} \mathrm{O}_{3}$ qui est favorisée, et ce au détriment de la couche intermédiaire (figure 8). La croissance de cette couche se fait à l'interface $\mathrm{Cr}_{2} \mathrm{O}_{3} / \mathrm{NiCr}_{2} \mathrm{O}_{4}$ et selon un mécanisme cationique comme en témoignent de nombreux pores présents à l'interface $\mathrm{Cr}_{2} \mathrm{O}_{3}$ /alliage. Comme à $600{ }^{\circ} \mathrm{C}$, l'application d'une contrainte externe en traction entraîne une augmentation de défauts dans la couche de $\mathrm{NiO}$ et favorise la diffusion de l'oxygène. En condition d'oxydation statique, $\mathrm{NiCr}_{2} \mathrm{O}_{4}$ se développe selon une réaction entre les précipités internes de $\mathrm{Cr}_{2} \mathrm{O}_{3}$, le nickel de l'alliage et l'oxygène, tant qu'une couche continue de $\mathrm{Cr}_{2} \mathrm{O}_{3}$ n'est pas formée. Une fois cette couche continue formée, la croissance des couches varie lentement. Sous contrainte, l'effet du temps ou de la déformation entraîne une modification de réaction interfaciale se traduisant par une déstabilisation de la structure spinelle et une extension de la couche d'oxyde de chrome à partir de l'interface $\mathrm{Cr}_{2} \mathrm{O}_{3} / \mathrm{NiCr}_{2} \mathrm{O}_{4}$.

\section{CONCLUSION}

L'ensemble des travaux présentés montre l'importance jouée par l'oxydation sur le comportement des matériaux. Un grand nombre de conséquences de l'oxydation sur les propriétés du matériau ou inversement de la nature du matériau sur les couches développées, conduisant à des modifications de propriétés physiques ou mécaniques. En fonction du type de défauts résultant et des modifications microstructurales et chimiques, l'oxydation peut avoir un effet soit adoucissant, soit renforçant sur certaines propriétés mécaniques (limite d'élasticité, allongement, fluage). Ces effets sont particulièrement sensibles dans le cas de produits minces (importance relative des zones modifiées par rapport à l'épaisseur du matériau) ou en pointe de fissures.

De nombreuses études traitent de fonctionnalisation de la surface ou de la zone externe du matériau (actions sur la microstructure ou la chimie au travers de traitements thermiques appropriés) pour que ce 
dernier satisfasse à une oxydation maîtrisée (cas des éléments dits actifs, effet d' écrouissage ....). Ces actions sont indispensables pour assurer une protection optimale du matériau à protéger. Il est toutefois important de vérifier et de ne pas négliger les conséquences de ces traitements haute température sur les modifications engendrées dans le substrat.

\section{Références}

[1] Graham M.J., Hussey R.J. and Cohen M.J., Electrochemical Soc., 120 (1973) 1523.

[2] Khoi N.N., Smeltzer W.W. and Embury J.D.J., Electrochemical Soc., 122 (1975) 1495.

[3] Molins R. and Andrieu E., Microscopy of Oxidation 2, edited by Newcomb and Bennett, The Institute of Materials, (1993) 162.

[4] Chattopadhyay B. and Wood G.C., Oxidation of Metals, 2 (1970) 373.

[5] Wood G.C., right I.G., Hodgkiess T. and Whittle D.P., Werkst. und Korrosion, 21 (1970) 900.

[6] Giggins C.S. and Petit F.S., Transactions of the metallurgical society of AIME, 245 (1969) 2509.

[7] Moulin P., Armanet F., Béranger G. and Lacombe P. Mém, Scient. Revue de Met., (1977) 143.

[8] Pieraggi B. and Dabosi F., Werkstoffe und Korrosion, 38 (1987) 584.

[9] Rouzou I., Molins R., Rémy L. and Jomard F., Materials Science Forum, 461-464 (2004) 101.

[10] Salmon C., Tiberghien D., Molins R., Colin C. and Delannay F., Materials at High Temperatures, 17, (2000) 271.

[11] Hales R. and Hill C., Corrosion Science, 12 (1972) 843.

[12] Harris J.E., Acta Metallurgica, 26 (1978) 1033.

[13] Péraldi R., Monceau D. and Pieraggi B. Oxid. Met. 58 (2002) 249.

[14] Huntz A.M., Andrieux M. and Molins R., Mat. Sc. Eng. A, 415 (2006) 21.

[15] Huntz A.M., Andrieux M. and Molins R., Mat. Sc. Eng. A, 417 (2006) 8.

[16] Funkenbusch W., Smeggil J.G. and Bornstein N.S., Metall. Trans. A, 16 (1985) 1164.

[17] Molins R., Rouzou I. and Hou P., Materials Science and Engineering A (2006).

[18] Béranger G., Armanet F. and Lambertin M., in Role of active elements in the oxidation behaviour of high temperature metals and alloys, ed. E. Lang, Elsevier, 33 (1989).

[19] Bennett M.J. and Moon D.P. in Role of active elements in the oxidation behaviour of high temperature metals and alloys, ed. E. Lang, Elsevier, 111 (1989).

[20] Stott F.H., Mat. Science Forum, 251-254 (1997) 19.

[21] Hou P., Chia V. and Brown I., Surface and Coat. Technology, 51 (1992) 73.

[22] Pint A., Oxid. Met., 45 (1996) 1.

[23] Messaoudi K., Huntz A.M. and Lesage B., Mat. Science Eng., A 247 (1998) 248.

[24] Maréchal L., Lesage B., Huntz A.M. and Molins R., Oxidation of Metals, 60 (2003) 1.

[25] Merceron G., Molins R. and Strudel J.L., Materials at High Temperatures, 17 (2000) 149.

[26] Quadakkers W.J. and Bongartz K., Werkstoffe und Korrosion, 45 (1994) 232.

[27] Schmitt J.F., Pacia N., Pigeat P. and Weber B., Oxidation of Metals, 44 (1995) 429.

[28] Flower H.M. and Wilcox B.A., Corrosion science, 17 (1977) 253.

[29] Calvarin G., Molins R. and Huntz A.M., Oxidation of Metals, 53 (2000) 25.

[30] Calvarin G., Molins R. and Huntz A.M., Oxidation of Metals, 54 (2000) 399. 
